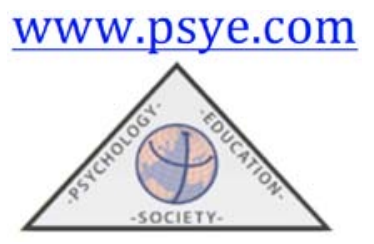

(C) Psychology, Society, \& Education, 2017. Vol. 9(1), pp. 71-87

ISSN 2171-2085 (print) / ISSN 1989-709X (online)

\title{
Instrucción directa con alumnado de Educación Primaria en riesgo de dificultades en el aprendizaje de las matemáticas
}

\author{
José I. NAVARRO GUZMÁN, María José NAVARRO CECILIA y Inmaculada \\ MENACHO \\ Universidad de Cádiz, España
}

(Recibido 25 Julio, 2016; Aceptado 16 Septiembre, 2016)

RESUMEN: La instrucción directa se ha utilizado como procedimiento de intervención para el aprendizaje de las matemáticas. Asimismo, estos procedimientos se han centrado tanto en los componentes de dominio general como de dominio específico. En este trabajo se estudiaron los efectos que sobre la competencia matemática temprana y la memoria de trabajo tuvieron diferentes programas de entrenamiento matemático, combinando actividades específicas de memoria de trabajo y de competencia matemática, a partir de actividades de instrucción directa, en niños de primero de educación primaria que presentaban riesgo de tener dificultades de aprendizaje de las matemáticas. Catorce participantes fueron identificados como alumnado en riesgo de tener dificultades de aprendizaje matemático mediante el test TEMT-i y asignados a un procedimiento pedagógico de intervención individualizado. A partir de un diseño cuasi experimental con dos medidas de las variables dependientes, los resultados mostraron que el programa de intervención promovió una clara mejora tanto en la competencia matemática, como en la memoria de trabajo.

Palabras clave: educación primaria, competencia matemática temprana, instrucción directa, memoria de trabajo, TEMT-i.

\section{Direct instruction to Primary Education students at risk of mathematics learning difficulties}

\begin{abstract}
Direct instruction has been used as a current intervention procedure for learning mathematics. In addition, these procedures have focused both components of general domain as specific domain. In this paper the effects on early mathematical competence and working memory had different programs mathematical training was studied. The programs combined specific activities of working memory and math competence using a direct instructional procedure. Participants were first grade primary education at risk of having mathematics learning difficulties. Fourteen students were identified as at risk of mathematical learning difficulties by ENT-Rand assigned to an individualized educational intervention procedure. A quasi-experimental design with two dependent variables' measures was used. Results showed that the intervention program promoted a strong improvement in both mathematical competence, and working memory.
\end{abstract}

Keywords: primary education, early math competency, direct instruction, ENT-R, working memory

${ }^{1}$ Correspondencia: Departamento de Psicología, Universidad de Cádiz, 11510 Puerto Real-Cádiz, España Tel.: +34-956 016563; fax: +34-956 016419 Email:jose.navarro@uca.es 
El alumnado en riesgo de tener dificultades de aprendizaje de las matemáticas (DAM) suele manifestar estas dificultades en diferentes áreas. Se ha informado en diferentes estudios que las DAM incluyen déficits en los conceptos numéricos tempranos, en la combinación de números o en su computación (Jitendra, Pulles, Kiss, \&Houseworth, 2016). Las matemáticas suelen ser también difíciles para aquellos niños que han mostrado dificultades en las llamadas habilidades de dominio general, tales como la memoria de trabajo (Aragón, Navarro, Aguilar, Cerda, \& García-Sedeño, 2016), lenguaje, o atención (Fuchs et al., 2014). Por ello los profesores precisan de herramientas que identifiquen formas de enseñar basadas en la evidencia para la mejora del aprendizaje matemático.

Buena parte de la literatura sobre los procedimientos de intervención en DAM se ha centrado en las operaciones básicas, probablemente debido a la naturaleza jerárquica del desarrollo de las habilidades matemáticas (Codding, Shiyko, Russo, Birch, Fanning, \&Jaspen, 2007). El dominio de las habilidades básicas de computación es importante por varias razones y se requiere destreza en el cálculo para el éxito vida cotidiana independiente. En segundo lugar, estas habilidades pueden servir como base para las aplicaciones relacionadas con la administración de dinero y tiempo, así como el pensamiento abstracto y la resolución de problemas. Esto ha sido plasmado en muchos de los programas de aprendizaje matemático en la enseñanza primaria (NCTM, 2006). Una creciente línea de trabajo ha mostrado que diferentes intervenciones sencillas son eficaces para mejorar el cálculo con fluidez, incluyendo la temporización explícita (ET) (Rhymer, Skinner, Jackson, McNeill, Smith, \& Jackson, 2002) y Cubrir-Copiar-Comparar (CCC) (Poff, McLaughlin, \& Derby, 2012), o la instrucción directa con flash cards (FC) (Skinner, McLaughlin, y Logan, 1997; Navarro-Cecilia \& Navarro, 2015).

El método ET es un procedimiento que requiere que el alumnado vaya registrando su progreso en intervalos de tiempo establecido cuando finaliza una tarea. De este modo se les proporciona una retroalimentación de la cantidad de problemas que ha completado (Rhymer, et al., 2002).

El procedimiento Copiar, Cubrir y Comparar (CCC) ha sido empleado para diferentes tareas de aprendizaje matemático en alumnado de educación primaria (Poncy, Skinner, \& Jaspers, 2007). Es un tipo de intervención académica que proporciona numerosas oportunidades de respuesta y retroalimentación inmediata. Sugiere que el alumnado mire la operación, la escriba mientras dice cada componente de la misma en voz alta o en silencio, cubra la operación, la escriba de nuevamente de memoria y compare la operación escrita con el modelo establecido, para comprobar si la ha realizado bien. Si la operación matemática fue incorrecta se corrige el error (Cieslar, McLaughlin, \& Derby, 2008). Los resultados globales de numerosos estudios muestran una mejora de las tareas matemáticas con menos errores, siendo más eficiente si se ajusta la corrección de errores (Becker, McLaughlin, Weber, \&Gower, 2009).

Las flash cards (FC) son tarjetas que por un lado pueden tener un símbolo, imagen, número, frase, pregunta y por el otro tienen la respuesta o el significado. Se usan ampliamente para aprendizaje de conceptos sencillos y para facilitar la memorización de ciertas tareas. Se trata de un procedimiento de instrucción directa en el que el alumnado recibe retroalimentación con un componente de corrección de errores, de manera que no practican una habilidad 
incorrectamente. Este método de instrucción directa ha tenido éxito en la enseñanza de las habilidades académicas de los niños con necesidades especiales (Hayter, Scott, McLaughlin, \& Weber, 2007).

Otros métodos de instrucción directa que vienen utilizándose en la educación primaria parten de una perspectiva de entender que el desarrollo matemático está basado en el pensamiento lógico, la enseñanza del sistema de numeración convencional y el aprendizaje significativo y contextualizado de los contenidos matemáticos (Aguilar, Aragón, \& Navarro, 2015; Bryant \&Nunes, 2002). Así mismo esta idea se complementa con una perspectiva más interaccionista que indica que las operaciones piagetianas y el conteo no tienen por qué ser separados y que juntos contribuyen al desarrollo del número (Araujo, Aragón, Aguilar, Navarro, \& Ruiz, 2014). Con este enfoque, el desarrollo del número es reformulado por el constructo denominado competencia matemática temprana. El concepto de competencia en el área de las matemáticas implica la habilidad de entender, juzgar, hacer y usar las matemáticas en una variedad de situaciones y contextos intra y extra matemáticos, en los que éstas podrían jugar un rol. Dichas habilidades pueden tener diversos niveles de complejidad, pues dependen de los sujetos que las poseen y del modo en que son movilizadas como respuesta a las demandas del entorno y situaciones específicas. En esta dirección va alguno de los programas de intervención que ha servido para el desarrollo de este trabajo empírico.

Las diferencias de rendimiento en el desempeño matemático pueden deberse a muy diversas causas (Aragón, Delgado, Aguilar, Araújo, \& Navarro, 2013). La investigación reciente sugiere que existen dos tipos de factores predictores del rendimiento matemático. Los predictores de dominio general (Passolunghi, Lanfran-chi, Altoè, y Sollazzo, 2015), como, por ejemplo, la inteligencia general y la memoria de trabajo. Y los de dominio específico tales como las habilidades de conteo en matemáticas (De Smedt, Janssen, Bouwens, Verschaffel, Boets, \&Ghesquière, 2009). Esta diversidad de factores determinantes de las habilidades matemáticas es fundamental para llegar a explicar el hecho de que algunos alumnos tengan importantes dificultades en matemáticas a pesar de manifestar un óptimo funcionamiento en los predictores de dominio general (Landerl, Bevan, \&Butterworth, 2004). Dentro de los componentes de dominio general, la memoria de trabajo (MT) ha sido un proceso particularmente interesante para la investigación reciente en cognición matemática (Passolunghi et al., 2015). En este sentido también se ha focalizado el campo de intervención educativo en el desarrollo de programas dirigidos a entrenar la MT específicamente o en paralelo al entrenamiento de otros predictores de dominio específico (Ramirez, Gunderson, Levine, \&Beilock, 2013). Sin embargo, las relaciones entre dificultades matemáticas y MT no están completamente clarificadas. Algunos autores han indicado que aquellos alumnos con peor rendimiento en matemática temprana mostraron también un bajo rendimiento en MT (Toll, Van der Ven, Kroesbergen, \& Van Luit (2011). Sin embargo, otros han relacionado malos ejecutores matemáticos con buenos resultados en MT (Passolunghi et al., 2015).

\section{Propósito de este estudio}

La intervención temprana con actividades numéricas en niños que presentan dificultades aritméticas puede mejorar su rendimiento de forma significativa. Por ello, el objetivo de este estudio fue proporcionar diferentes métodos de instrucción directa en 14 alumnos de 6 años que 
mostraban un alto riesgo de presentar dificultades de aprendizaje de las matemáticas. Se trata asimismo de comprobar la eficacia de estos programas de entrenamiento mediante instrucción directa, con base en los resultados obtenidos en diferentes pruebas de competencias matemáticas y de memoria de trabajo.

\section{Método}

\section{Participantes}

Los participantes fueron 50 alumnos, de los cuales 23 eran niños y 27 niñas, que cursaban el último nivel de Educación Infantil y con edades comprendidas entre los 5 y 6 años $(\mathrm{M}=5.24 ; \mathrm{DT}=.43)$ en el momento de la evaluación inicial.

El alumnado pertenecía a un centro escolar de carácter público situado en una población inferior a los 100.000 habitantes. El centro fue seleccionado de manera incidental de forma que su localización no pusiera impedimentos a la hora de implementar la intervención, y porque en todo momento existió una óptima colaboración por parte de las familias y del profesorado del centro. Se ha tenido en cuenta el nivel socio-económico y cultural de las familias y del área urbana en el que se encontraba el centro, que recibe alumnado de un nivel socioeconómico medio y medio-bajo y cuyas familias se veían afectadas en su mayoría por la problemática del desempleo. Ninguno de los participantes mostraba trastornos del desarrollo, ni déficits sensoriales, cognitivos o necesidades educativas especiales resultantes de motivos socioculturales.

Tras la evaluación de todos los participantes con la versión española del test de evaluación de la competencia matemática temprana-informatizado (TEMT-i) (Van Luit et al., 2015), aquellos que obtuvieron una puntuación inferior a 20 puntos (de un total de 45 puntos posibles) fueron seleccionados para la puesta en marcha del programa de intervención. Así, del total de los participantes, 14 estudiantes (6 niños y 8 niñas) de $1^{\circ}$ de Educación Primaria, con edades de entre 6 y 7 años, fueron quienes llevaron a cabo el entrenamiento.

\section{Instrumento}

Se utilizaron dos instrumentos de medida: uno para la evaluación de la competencia matemática temprana (CMT) y otro para la MT.

Test de evaluación de la competencia matemática temprana-informatizado (TEMT-i) (Van Luit et al., 2015). Esta prueba evalúa nueve componentes de la competencia matemática temprana (CMT): Comparación, clasificación, correspondencia uno a uno, seriación, conteo verbal, conteo estructurado, conteo resultante (sin señalar), conocimiento general de los números y Estimación. Dirigida a grupos de edad de 4 a 7 años, está constituida por tres versiones psicométricamente paralelas (A, B y C) de 45 ítems cada una, presentando una puntuación directa máxima de 45 puntos (un punto por cada ítem correcto). La prueba se administra individualmente a través de computadora y tiene un tiempo promedio de ejecución de 30 minutos. El TEMT-iobtuvoun alpha de Cronbach de .92. 
Automated Working Memory Assessment (AWMA) (Alloway, 2007).Es una herramienta ampliamente usada para la evaluación de la memoria. Esta batería se administra por ordenador de manera individualizada y está compuesta por 12 tareas que permiten evaluar todos los aspectos del sistema de memoria. Las instrucciones y los estímulos verbales son presentados de forma auditiva, excluyendo así los requisitos propios de la lectura. De esta batería se han administrado las tareas de recuerdo de dígitos en orden inverso (back Ward digit recall) y figura diferente (Odd One Out) por medir la capacidad de memoria de trabajo verbal (ejecutivo central) y viso-espacial, respectivamente.

- Recuerdo de dígitos en orden inverso (back Ward digit recall). Su objetivo es la evaluación de la memoria de trabajo verbal. Se presentan secuencias de números cada vez más amplias y el niño tiene que recordarlas y nombrarlas en el orden inverso. Está formada por 6 bloques de 6 ítems cada uno, siendo así la amplitud máxima de 6 dígitos. Su alpha de Cronbach fue de .89 .

- Figura diferente (Odd One Out). Su objetivo es la evaluación de la memoria de trabajo viso-espacial. Se muestran conjuntos de tres figuras en la pantalla de las cuales una de ellas es diferente a las otras dos. El evaluado tiene que indicar cuál es la diferente y una vez que se han presentado un cierto número de conjuntos, también debe señalar el lugar en el que apareció en el orden exacto. Consta de 7 bloques de figuras con 6 ítems cada uno. Su alpha de Cronbach fue de .91.

La administración de las pruebas se realizó en buenas condiciones de evaluación por parte de los autores y otra persona especializada en la evaluación psicológica y educativa. En todos los casos se evaluó a los participantes durante el horario escolar, respetando los descansos del alumnado.

\section{Descripción del programa de intervención}

(1) Programa de instrucción directa de dominios matemáticos específicos. Mediante el software Superlab 4.5. se generaron listados de actividades para el entrenamiento de las siguientes competencias matemáticas: asociación de cantidades con su grafía y con patrones regulares de puntos (subitización), conceptos de correspondencia uno a uno, comparación de cantidades, tareas de clasificación y seriación, conteo estructurado y resultante, sumas y restas simples, estimación en la recta numérica. El programa genera un total de 30 estímulos en una pantalla de ordenador y el alumno debe responder en un teclado o bien señalando la respuesta correcta en la pantalla, o respondiendo verbalmente a las preguntas-estímulo. Por ejemplo, para una tarea de estimación el alumno debía responder señalando con el dedo en la pantalla (ver figura 1) a la siguiente pregunta: "une el número de arriba con su lugar correspondiente en el tendedero de números. Fíjate que esta vez va del 0 al 20”. 


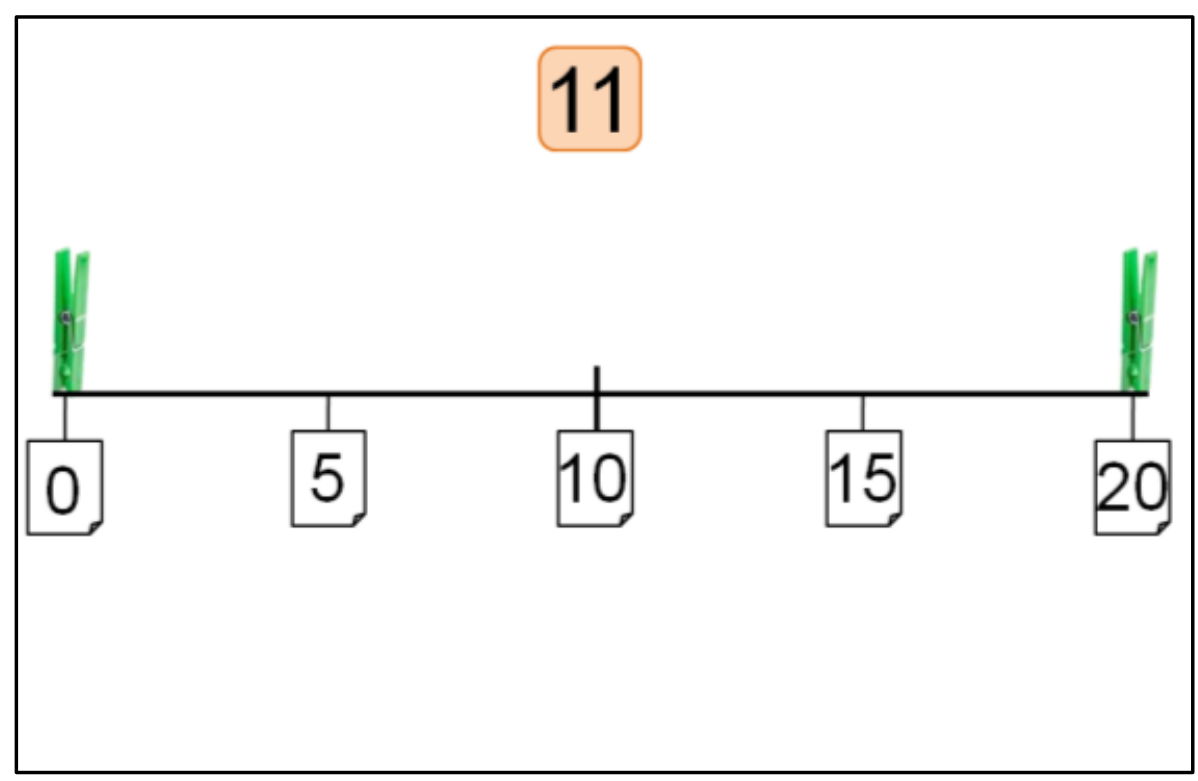

Figura 1. Modelo de ítem de estimación.

(2) Programa de instrucción directa con material manipulativo

2.1. Tendedero de números: diseñado para trabajar didácticamente con la recta numérica, esta actividad manipulativa consistía en una especie de tendedero de ropa hecho con madera (semejante al de la figura 1), en el que se invitaba individualmente a los niños a posicionar una tarjeta numérica (tarjeta de cifras) y a sujetarla en la cuerda con la mayor precisión espacial posible dentro de "la recta numérica”. A la izquierda del tendedero se situaba un número cualquiera $(0,10, \ldots)$ y, en el extremo opuesto, otro de mayor magnitud $(20,15$, ...), debiendo estimar la posición de un número dado en la línea. Las cifras iban desde el 0 hasta el 50, y se les presentaba en dificultad creciente.

2.2. Fichas de conteo, patrones de dedos y secuencia numérica: Se realizaron secuencias de ejercicios sobre conteo, secuencia numérica y patrones de dedos, con el fin de conseguir una adecuada destreza sobre estos aspectos. Las actividades dentro de este programa fueron las siguientes:

2.2.1. Dibujos escondidos: con números del 0 al 50 (como máximo), había dibujado una figura sobre un papel (caballo, casa...), donde los niños podían poner en práctica sus habilidades de conteo y secuencia numérica para formar un dibujo uniendo los números de manera consecutiva.

2.2.2. Seriación: consistían en completar los huecos que faltan en diversas series de números consecutivos escritos en un papel, tanto hacia delante como hacia atrás, y empezando o terminando en cualquier cifra hasta 50. 
2.2.3. Asociación: los participantes tenían que unir con líneas las grafías de los números del 0 al 9 y su cantidad, reflejada en los patrones de dedos dibujados en un papel (figura 2).

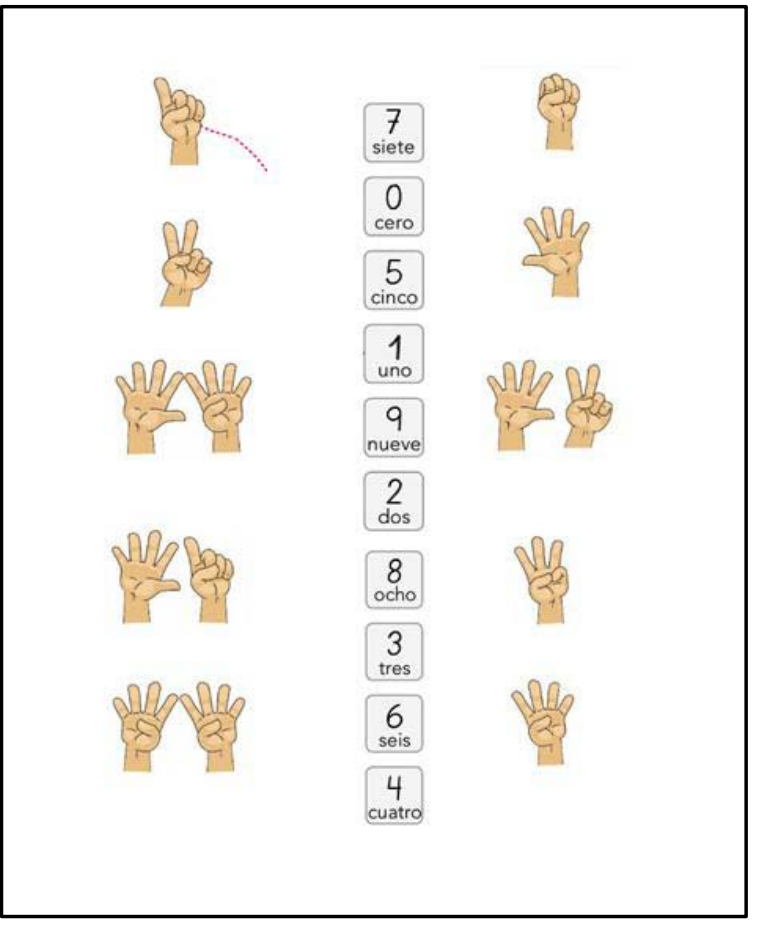

Figura 2. Ejemplo de ítem mostrando patrones de dedos.

(3) Programa específico de intervención en MT. Se elaboraron 91 actividades específicas a través del programa de presentación Microsoft Power Point, con distintas tareas matemáticas. Los 91 estímulos contenían diferentes tipos de ítems desarrollados en dificultad progresiva, en las que los estímulos eran presentados visualmente. Las instrucciones se proporcionaban de forma oral por parte de la profesora, tratando de suscitar una respuesta verbal en el alumno. Las actividades diseñadas fueron:

3.1. Recuerdo inmediato de animales (10 ítems): se les presentaba a los niños una diapositiva con animales y, aproximadamente, 5 segundos después, otra en blanco. A continuación, se les pedía recordar el nombre del animal que era más grande o más pequeño, aumentando la dificultad a los dos más grandes y los dos más pequeños de forma progresiva. La amplitud variaba de 3 a 4 animales.

3.2. Recuerdo de cantidades (8 ítems): los alumnos tenían que contar un cierto número de elementos presentados como estímulos en la pantalla, algunos de ellos seguían patrones espaciales y otros estaban dispuestos de forma desorganizada. A continuación, se presentaba una diapositiva con varios dados con distintos números de puntos. Los participantes debían señalar el dado que tenía el mismo número de puntos que objetos presentados en el anterior estímulo. Por ejemplo: Estímulo 1, “Cuenta estas piruletas. ¿Cuántas había?”; Estímulo 2, 
“Señala el recuadro con los dados que tengan el mismo número de puntos” (figura 3)

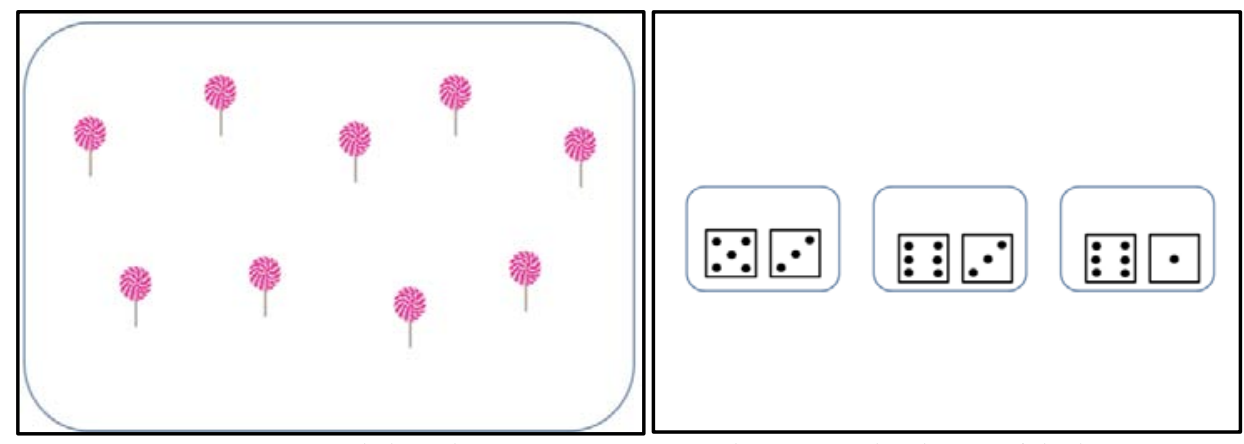

Figura 3. Modelos de ítem (E1 y E2) de recuerdo de cantidades

3.3. Amplitud de Contar (a partir de Case, Kurland, \& Goldberg, 1982): se le presentan al niño estímulos en forma de cuadrados o tarjetas con patrones regulares de puntos negros (similar a las caras de un dado). Estos puntos negros debían ser contados y retener los resultados del recuento. A continuación, aparece un estímulo en blanco y el estudiante debe repetir mediante recuerdo serial la cantidad de puntos de cada tarjeta de la serie. La tarea se inicia presentando una serie de dos tarjetas, usándose el mismo procedimiento con las series de tres (el estudiante debe recordar cada vez tres números) y cuatro tarjetas, hasta que es incapaz de recordar en orden serial los números de una misma amplitud. Había un total de cuatro series para la amplitud 2 y 3, y dos series de 4 tarjetas.

3.4. Amplitud de lectura de números: este tipo de tareas se basó en la Prueba de Amplitud Lectora de Daneman \& Carpenter (1983). Durante la tarea, el niño leía una secuencia de números en voz alta y indicaba si la secuencia era correcta o no (los números presentados beben de ser consecutivos). Seguidamente, aparece un nuevo estímulo consistente en una pantalla de ordenador en blanco y se le pedía recordar cuál era el último número de cada secuencia, en el mismo orden que las ha leído. Se elaboraron un total de tres tareas con dos y tres secuencias cada una, manteniendo la amplitud en 3.

3.5. Recuerdo serial de dígitos en orden inverso: se presentaban secuencias orales de dígitos (de 2 hasta 5), que debían ser recordados inmediatamente en orden inverso. Estas actividades consistieron en estímulos de $6,12,8$ y 4 secuencias para cada amplitud, respectivamente.

\section{Procedimiento}

Al inicio de la investigación se solicitó el consentimiento informado de los padres de los participantes, además del permiso del profesorado y directivos del centro escolar donde se desarrolló el trabajo, cumpliendo escrupulosamente los criterios éticos exigidos para este tipo de investigación educativa. Padres, alumnado y profesores mostraron en todo momento una 
actitud colaboradora hacia el estudio. Se realizaron varias reuniones explicativas del proyecto con los responsables escolares y, al finalizar el mismo, se emitió un informe de los resultados obtenidos de los escolares. El estudio fue desarrollado en cuatro fases diferenciadas.

Fase 1. Evaluación pre- de los participantes: Se efectuó la recogida de datos pretest ente los meses de marzo y abril de 2014, donde se administraron el TEMT-i para medir la CMT y la batería AWMA para evaluar la MT. El proceso de recogida de datos se llevó a cabo en dos sesiones de evaluación para cada participante, evaluándose así en una de ellas la CMT y en la otra la MT, cuyos tiempos de duración fueron de aproximadamente 30 minutos cada una. Ambas se aplicaron de forma individual, donde los alumnos utilizaban un ordenador con ratón óptico.

Fase 2. Selección del grupo de riesgo de padecer DAM. Una vez evaluada la CMT, se seleccionaron aquellos participantes que obtuvieron una puntuación inferior a 20 puntos (de un total de 45 puntos posibles). Esta selección se realizó al inicio del primer curso de Educación Primaria. Este criterio de corte está especificado en la interpretación del TEMT-i, por encontrarse en un nivel de respuesta una desviación típica por debajo del valor medio de la edad. De esta forma, del total de 50 niños y niñas evaluados, se seleccionaron 14 que formarían el grupo de tratamiento.

Fase 3. Intervención educativa especializada. En esta fase se implementó el programa de intervención con el grupo de tratamiento tanto en habilidades matemáticas como en MT. Se aplicaron los procedimientos descritos en el apartado anterior: (1) programa de instrucción directa de dominios matemáticos específicos; (2) programa de instrucción directa con material manipulativo; y (3) programa específico de intervención en MT. Todas las sesiones de los programas de instrucción directa y MT fueron implementadas por una de las autoras de este trabajo y se llevó a cabo en pequeños grupos de 2 alumnos, o bien individuamente. La secuencia de intervención fue la siguiente:

(1) Programa de conteo, patrones de dedos y secuencia numérica. Desarrolladas con dos niños simultáneamente durante 12 sesiones de 10 minutos cada una.

(2) Tendedero de números y patrones de puntos. Desarrolladas con dos niños simultáneamente durante 11 sesiones de 15 minutos.

(3) Programa específico de entrenamiento de la MT. Desarrolladas durante 22 sesiones de 15 minutos. Realizaron diversas actividades en el ordenador a través de la herramienta diseñada en Microsoft Power Point. En sesiones individuales, se les iba mostrando los diferentes tipos de ítems, proporcionándoles las instrucciones de forma oral. El alumnado debía facilitar una respuesta verbal en todas las tareas, además de señalar la pantalla en los casos en que se requería. En cada sesión se trabajaba 45 ítems con este procedimiento.

Fase 4. Evaluación post-. Una vez finalizadas las sesiones de intervención, se llevó a cabo una evaluación post- en la que se administró la versión B del test TEMT-i y la batería AWMA de nuevo, con el fin de analizar la eficacia del programa de intervención implementado y el efecto de dicha intervención en los alumnos. Esta segunda evaluación se realizó en las mismas condiciones de espacio y tiempo que en la fase 1.

Las sesiones de evaluación e intervención tuvieron lugar dentro del centro escolar al que pertenecían los alumnos, en la misma franja horaria, en una sala específica para ello, con 
buenas condiciones de luz y habitabilidad, idóneas para la intervención pedagógica, y diferente al aula ordinaria con el fin de evitar ruidos y distracciones, además de realizarse de forma individual o por parejas, respectivamente.

\section{Análisis de datos}

Se empleó un diseño cuasi experimental con medidas pre y post de las variables dependientes (TEMT-i y AWMA), para comprobar la influencia del programa de intervención. Inicialmente se realizó un cálculo de los estadísticos descriptivos de todas las variables evaluadas con el TEMT-i y el AWMA. Asimismo, se realizó la prueba de contrastes de hipótesis de Kolmogórov-Smirnov para conocer si las variables se distribuían de forma normal. Para comprobar que las diferencias encontradas antes y después del programa de intervención se seleccionó la prueba de Wilcoxon basada en las diferencias en valor absoluto de las puntuaciones obtenidas en cada condición (pretest y postest) y posteriormente se calculó el tamaño del efecto a través de la $d$ de Cohen.

\section{Resultados}

Tabla 1. Estadísticos descriptivos de los resultados obtenidos en el TEMT-i y AWMA en la fase previa a

\begin{tabular}{|c|c|c|c|c|c|}
\hline & Media & $\begin{array}{l}\text { Desviación } \\
\text { Típica }\end{array}$ & $\begin{array}{l}\text { Valor } \\
\text { mínimo }\end{array}$ & $\begin{array}{l}\text { Valor } \\
\text { máximo }\end{array}$ & Varianza \\
\hline TEMT-i Total test & 21.14 & 5.96 & 10 & 35 & 35.5 \\
\hline TEMT-i Total relacional & 11.67 & 2.56 & 6 & 16 & 6.5 \\
\hline TEMT-i Total numérico & 9.47 & 4.45 & 2 & 19 & 19.8 \\
\hline $\begin{array}{l}\text { AWMA } \\
\text { (BackwardDigitRecall) }\end{array}$ & 103.38 & 10.24 & 82 & 130 & 104.8 \\
\hline AWMA (Odd One Out) & 105.52 & 15.17 & 79 & 131 & 230.1 \\
\hline
\end{tabular}

La administración de las pruebas AWMA y TEMT-i en la fase inicial a 50 participantes permitió conocer los niveles de MT y de competencia matemática temprana, así como poder identificar a aquellos estudiantes en riesgo de tener DAM. En la tabla 1 se presentan los estadísticos descriptivos de ambas pruebas.

Tabla 2. Frecuencias de casos y estadísticos descriptivos para los participantes con puntuación en el TEMT-i, menor a 20 puntos totales

\begin{tabular}{llll}
\hline Frecuencia & Puntuación & Estadísticos & \\
\hline 1 & 10 & Media & 13.7 \\
1 & 11 & Mediana & 14.5 \\
2 & 12 & Moda & 15 \\
2 & 14 & Desv. típ. & 2.0 \\
4 & 15 & Varianza & 4.0 \\
2 & 16 & Rango & 6 \\
1 & 18 & Mínimo & 10 \\
1 & 20 & Máximo & 16
\end{tabular}

(c) Psy, Soc, \&Educ, 2017, Vol. 9(1) 


\begin{tabular}{lll} 
Percentiles & 25 & 12.0 \\
& 50 & 14.5 \\
& 75 & 15.0 \\
\hline
\end{tabular}

Como se indicó en el apartado de procedimiento, de los 50 alumnos que fueron evaluados, 14 de ellos obtuvieron valores inferiores a 20 en la puntuación total del TEMT-i, que serían los seleccionados para el proceso de intervención al considerarlos en riesgo de tener DM. Sus datos descriptivos aparecen en la tabla 2.

Tabla 3. Resultados del cálculo realizado entre las puntuaciones pre y post obtenidas con el TEMT-i, antes (versión A) y después (versión B) de la intervención y con las subpruebas del AWMA, mediante el test de los rangos con signo de Wilcoxon

\begin{tabular}{llll}
\hline Comparaciones entre pruebas & $\mathrm{N}$ & $\mathrm{Z}$ & $\mathrm{p}$ \\
\hline TEMT-i (A)-TEMT-i (B) & 14 & -3.300 & .001
\end{tabular}

AWMA pre y post (Backward Digit Recall) $\quad 14 \quad-3.113 \quad .001$

AWMA pre y post (Odd One Out) $\quad 14 \quad-1.995 \quad 0.4$

A partir de aquí se trabajó con los 14 participantes a los que se les administró el programa de intervención con instrucción directa y entrenamiento en MT. Realizamos un contraste entre los valores obtenidos en el TEMT-i antes y después de la intervención, mediante la prueba no paramétrica de los rangos con signo de Wilcoxon, cuyos resultados fueron estadísticamente significativos $(Z=-3.300 ; \mathrm{p}<.001)$. Asimismo, se realizaron los contrastes con los valores de MT resultando también significativos $(\mathrm{Z}=-3.113 ; \mathrm{p}<.001 ; \mathrm{Z}=-3.113$, $\mathrm{p}$ $<.04$, respectivamente) (tabla 3). Se calculó el tamaño de efecto para la competencia matemática temprana a partir de los valores de $\mathrm{t}(13 \mathrm{gl})=-23.88(\mathrm{p}<.000)$, arrojando una d de Cohen $=.92$.

Tabla 4. Ganancias obtenidas tras el programa de intervención en el AUWMA y en el TEMT-i

\begin{tabular}{lllllllll}
\hline $\begin{array}{l}\text { BDR } \\
\text { Pre }\end{array}$ & $\begin{array}{l}\text { BDR } \\
\text { Post }\end{array}$ & $\begin{array}{l}\text { Incremento } \\
\text { en BD }\end{array}$ & $\begin{array}{l}\text { OOO } \\
\text { Pre }\end{array}$ & $\begin{array}{l}\text { OOO } \\
\text { Post }\end{array}$ & $\begin{array}{l}\text { Incremento } \\
\text { en OOO }\end{array}$ & $\begin{array}{l}\text { TEMT-i } \\
\text { Pre }\end{array}$ & $\begin{array}{l}\text { TEMT-i } \\
\text { Post }\end{array}$ & $\begin{array}{l}\text { Incremento } \\
\text { en TEMT-i }\end{array}$ \\
\hline 95 & 122 & 27 & 120 & 117 & -3 & 15 & 44 & 29 \\
104 & 132 & 28 & 115 & 129 & 14 & 16 & 43 & 27 \\
100 & 114 & 14 & 90 & 98 & 8 & 10 & 36 & 26 \\
108 & 119 & 11 & 95 & 102 & 7 & 15 & 41 & 26 \\
87 & 106 & 19 & 87 & 94 & 7 & 18 & 38 & 20 \\
113 & 119 & 6 & 131 & 113 & -18 & 15 & 43 & 28 \\
100 & 114 & 14 & 105 & 102 & -3 & 11 & 38 & 27 \\
& & & & & & & & \\
\end{tabular}




\begin{tabular}{lllllllll}
92 & 102 & 10 & 83 & 90 & 7 & 20 & 43 & 23 \\
104 & 119 & 15 & 120 & 121 & 1 & 14 & 39 & 25 \\
104 & 123 & 19 & 95 & 106 & 11 & 15 & 43 & 28 \\
91 & 114 & 23 & 85 & 106 & 21 & 12 & 28 & 16 \\
104 & 101 & -3 & 90 & 106 & 16 & 12 & 37 & 25 \\
104 & 119 & 15 & 95 & 106 & 11 & 14 & 35 & 21 \\
105 & 110 & 5 & 85 & 87 & 2 & 16 & 36 & 20 \\
\hline
\end{tabular}

BD: Backward Digit Recall; OOO: Odd One Out

Para conocer el nivel de ganancias obtenido por el alumnado participante en los programas se calculó las ganancias de cada uno de los alumnos en la evaluación post test del TEMT-i. Los resultados indicaron que en todos los casos mejoraron significativamente equiparándose en la mayoría de ellos a los que se considera fuera una adecuada competencia matemática con esta prueba (tabla 4).

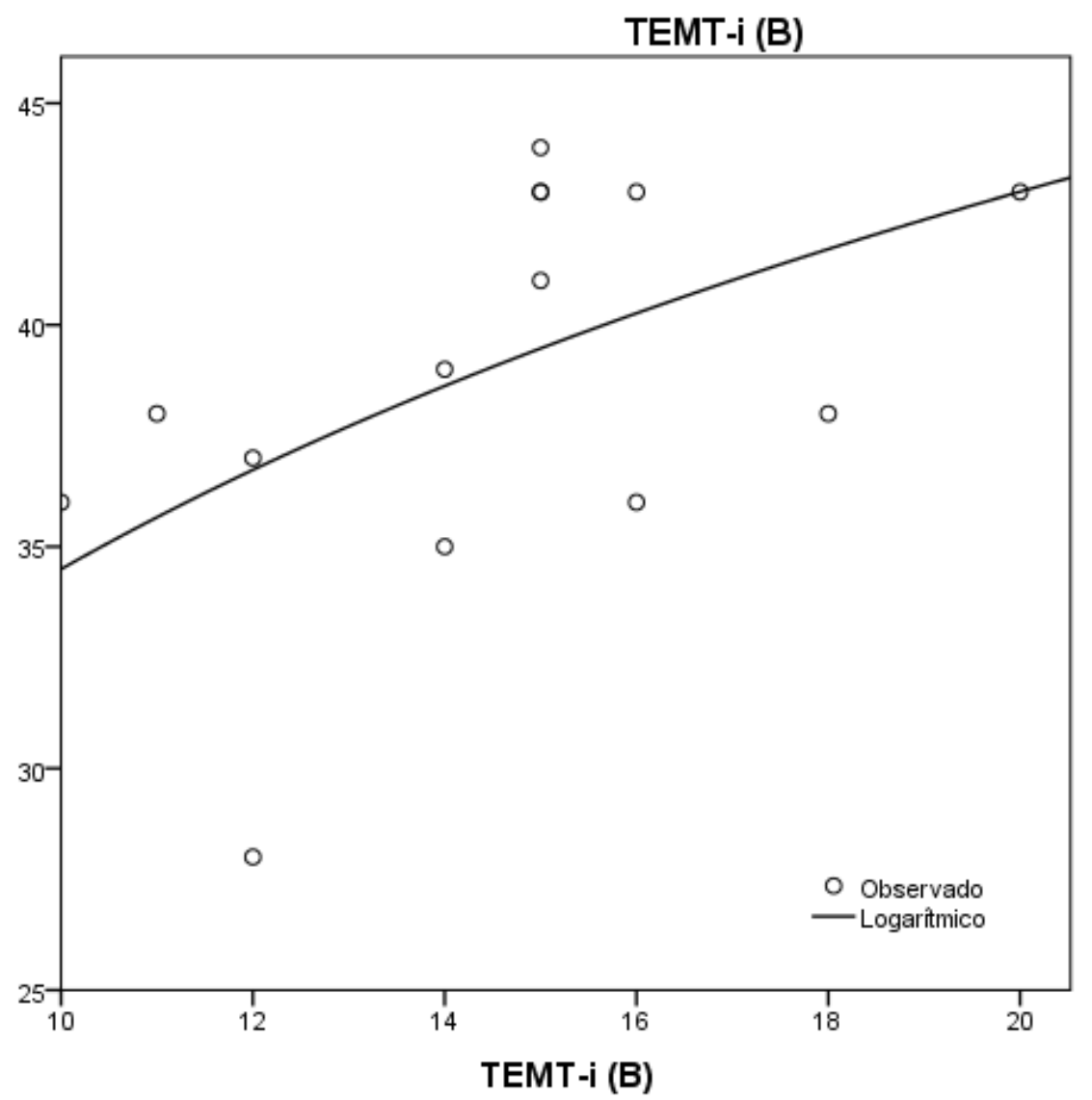

Figura 4. Modelo logarítmico mostrado por la evolución de los incrementos de la competencia matemática temprana evaluada por el TEMT-i. 
Los cambios en las ganancias obtenidas en la competencia matemática temprana muestran que tras la intervención todos los participantes mejoraron, de manera que 11 de los alumnos salieron de la zona de riesgo señalada por el TEMT-i. Las ganancias finales mostraron una función logarítmica generada por un modelo de $\mathrm{F}=4.502(\mathrm{p}<0.5)$, que puede visualizarse en la figura 4. Si comparamos la curva de frecuencia de los resultados del TEMT-i, versión A, con la versión B, como se muestra en la figura 5 , claramente se observa un desplazamiento de los valores hacia una posición más cercana a niveles de competencia matemática temprana adecuados según los parámetros facilitados por la prueba. Es decir, los alumnos entrenados superaron sus puntuaciones obtenidas en la versión A del TEMT-i.

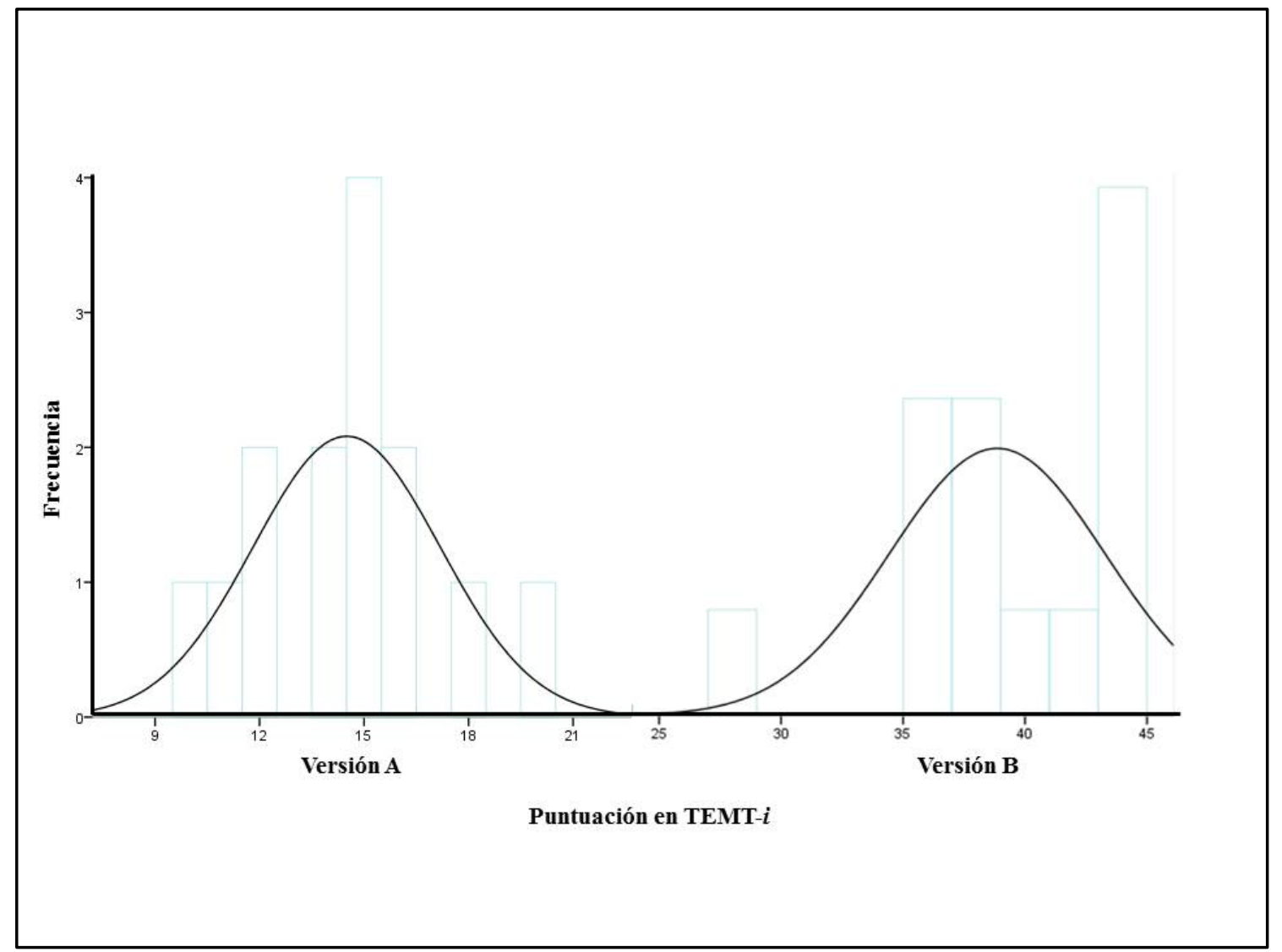

Figura 5. Distribución de frecuencias de los resultados obtenidos por los alumnos en las versiones A y B del TEMT-i.

\section{Discusión}

Los alumnos en el inicio de la edad primaria que muestran dificultades en su competencia matemática pueden estar en riesgo de arrastrar estas dificultades a lo largo de la escolarización. Se ha pretendido con este trabajo mostrar que un adecuado tratamiento en aspectos de dominio general (MT) y de dominio específico (competencia matemática), 
mediante programas puntuales de instrucción directa, pueden reducir el riesgo de DAM.

Desde nuestro punto de vista, encontramos que la instrucción directa ha tenido un fuerte impacto en el aprendizaje de conceptos relacionados con la competencia matemática temprana desarrollada en los primeros cursos de la Educación Primaria (comparación de cantidades, conteo, estimación, etc.) Efectivamente para 11 de los 14 alumnos intervenidos, el porcentaje de ganancias en el TEM-i fue claramente significativo, llegando a igualar en muchos casos a los demás alumnos que presentaban un valor inicial sin riesgo. Estos resultados son esperanzadores y corroboran trabajos anteriores realizados con otras metodologías diferentes. Butterworth, Varma, \&Laurillard (2011) informan que en el caso de los discalcúlicos, ha habido múltiples programas eficientes de muy diverso corte metodológico (Number Race, Graphogame-Maths, que son dos juegos adaptativos de ordenador), pero se necesitan estudios más a largo plazo para comprobar qué grado de mejora desarrollan en la educación secundaria. Por nuestra parte hemos elaborado un programa informático paralelo que resultó efectivo en el aprendizaje matemático temprana con instrucción asistida por ordenador (Aragón, RuizCagigas, Aguilar, Araujo, \& Navarro, 2013), con buenos resultados. La instrucción directa también ha resultado de interés en el campo educativo de las matemáticas con resultados en la misma dirección encontrada en este trabajo (Navarro-Cecilia \& Navarro, 2015; Poncy, Skinner, \& Jaspers, 2007). En consecuencia, entrenar aspectos de domino específico genera un efecto significativo en parámetros como el conteo, la estimación, la seriación o el cálculo simple.

El análisis de los cambios en los aspectos de dominio general (como es la MT) ha sido también muy satisfactorio en este estudio, aunque no tan contundente como la competencia matemática temprana. El análisis más pormenorizado de las dos tareas diferenciadas permite sacar conclusiones complementarias en la línea de la literatura especializada. La memoria de trabajo es una de las funciones ejecutivas más implicadas en la competencia matemática (Toll et al., 2011). Sin embargo, se trata de un concepto con múltiples funciones que se le ha relacionado con variedad de tareas académicas y de la vida cotidiana. Los dos aspectos considerados en este estudio han sido la memoria de trabajo verbal y la memoria de trabajo viso-espacial. En ambos casos se han conseguido resultados eficientes con el programa de intervención, si bien claramente la memoria de trabajo verbal (memoria de números recordados al revés) encontró una mayor significación estadística. La interpretación que hacemos de estos datos tiene relación con el material de entrenamiento desarrollado con el procedimiento de intervención directa. Si bien había mucho material manipulativo desplegado a lo largo de las sesiones del programa, la comunicación verbal fue constante con los niños, que además realizaron los programas de manera individualizada. Este tipo de actividades hace que los niños desplieguen un perfil característico de fortalecimiento de la MT. Las exigencias del método de enseñanza directo, trae consigo el desarrollo de habilidades de dominio general, que se ponen en marcha a la hora de implementar habilidades de dominio específico, propias del desempeño matemático. El alumnado fue entrenado con distinto material manipulativo y figurativo, menos abstracto. De esta manera, en tareas de cálculo implican que el alumnado opere de manera más eficiente con la memoria de trabajo, aplicando mentalmente las representaciones visoespaciales con las que han sido entrenados.

Sin embargo, algunos de los resultados necesitan confirmación a partir de trabajos que (c) Psy, Soc, \&Educ, 2017, Vol. 9(1) 
corrijan las limitaciones del actual. En este sentido, la elección de una muestra de manera incidental es un aspecto mejorable, así como el que el diseño de tipo cuasi experimental prescinda del grupo de control. Si bien los datos muestran claros cambios de las variables dependientes, el hecho de que los cambios sean intensos en relación a la competencia matemática temprana, la interpretación de los mismos debe hacerse con cautela, pues no se han podido controlar los aspectos relacionados con el desarrollo evolutivo propiamente dicho, o el efecto de la escolarización estándar. Otro de los aspectos de interés para futuras investigaciones sería conocer los efectos de doble interferencia del entrenamiento en competencia matemática y en memoria de trabajo. Si bien los cambios se han producido en ambos casos, conocer el peso de la interacción despejaría dudas sobre la importancia de los procesos de dominio general o específicos en el riesgo de padecer dificultades de aprendizaje de las matemáticas.

\section{Agradecimientos}

El trabajo pudo realizarse con la financiación parcial del proyecto I+D+i PSI201563856-P (MINECO/FEDER).

\section{Referencias}

Aguilar, M., Aragón, E., \& Navarro, J. I. (2015). Las dificultades de aprendizaje de las matemáticas (DAM). Estado del arte. Revista de Psicología y Educación, 10(2), 13-42.

Alloway, T. P. (2007). Automated working memory assessment. London: The Psychological Corporation.

Aragón, E., Ruiz-Cagigas, G., Aguilar, M., Araujo, A., \& Navarro, J.I. (2013). Jugando con números 2: El software complementario a la instrucción matemática temprana. Revista Internacional de Tecnología, Conocimiento y Sociedad, 2(2), 1-11.

Aragón, E. L., Delgado, I., Aguilar, M., Araújo, A., y Navarro, J. I. (2013). Estudio de la influencia de la inteligencia y el género en la evaluación matemática temprana. European Journal of Education and Psychology, 6(1), 5-18.

Aragón, E., Navarro, J. I., Aguilar, M., Cerda, G., \&García-Sedeño, M. (2016). Structural regression model of early math skills. Scandinavian Journal of Psychology, doi: 10.1111/sjop.12317 (on line first)

Araujo, A., Aragón, E., Aguilar, M., Navarro, J. I. \& Ruiz, G. (2014). Un estudio exploratorio para la adaptación de la versión española revisada del "EarlyNumeracy Test-R" para evaluar el aprendizaje matemático temprano. European Journal of Education and Psychology, 7(2), 83-93. doi: http://dx.doi.org/10.1989/ejep.v7i2.181

Becker, A., McLaughlin, T., Weber, K. P., \& Gower, J. (2009). The effects of copy cover and compare with and without additional error drill on multiplication fact fluency and accuracy. Electronic Journal of Research in Educational Psychology, 7(2), 747-760.

Bryant, P. \&Nunes, T. (2002). Children's understanding of mathematics. En U. Goswami (Ed.), Blackwell handbook of childhood cognitive development (pp. 412-439). Malden: Blackwell. 
Butterworth, B., Varma, S., \&Laurillard, D. (2011). Dyscalculia: from brain to education. Science, 332(6033), 1049-1053. doi: 10.1126/science.1201536

Case, R., Kurland, D. M., \& Goldberg, J. (1982). Operational efficiency and the growth of short-term memory span. Journal of Experimental Child Psychology, 33(3), 386-404.

Cieslar, W., McLaughlin, T. F., \& Derby, K. M. (2008). Effects of the copy, cover, and compare procedure on the math and spelling performance of a high school student with behavioral disorder: A case report. Preventing School Failure: Alternative Education for Children and Youth, 52(4), 45-52. doi:10.3200/PSFL.52.4.45-52

Codding, R. S., Shiyko, M., Russo, M., Birch, S., Fanning, E., \&Jaspen, D. (2007). Comparing mathematics interventions: Does initial level of fluency predict intervention effectiveness? Journal of School Psychology, 45(6), 603-617. doi: http://dx.doi.org/10.1016/j.jsp.2007.06.005

Daneman, M., \& Carpenter, P. A. (1983). Individual differences in integrating information between and within sentences. Journal of Experimental Psychology: Learning, Memory, and Cognition, 9(4), 561. doi:10.1037/0278-7393.9.4.561

De Smedt, B., Janssen, R., Bouwens, K., Verschaffel, L., Boets, B., \&Ghesquiere, P. (2009). Working memory and individual differences in mathematics achievement: A longitudinal study from first grade to second grade. Journal of Experimental Child Psychology, 103, 186-201. doi: http://dx.doi.org/10.1016/j.jecp.2009.01.004

Fuchs, L. S., Schumacher, R. F., Sterba, S. K., Long, J., Namkung, J., Malone, A., . Changas, P. (2014). Does working memory moderate the effects of fraction intervention? An aptitude-treatment interaction. Journal of Educational Psychology, 106, 499-514. doi: 10.1037/a0034341

Hayter, S., Scott, E., McLaughlin, T. F., \& Weber, K. P. (2007).The use of a modified direct instruction flashcard system with two high school students with developmental disabilities. Journal of Developmental and Physical Disabilities, 19, 409-415. doi: 10.1007/s10882-007-9059-3

Jitendra, A. K., Nelson, G., Pulles, S. M., Kiss, A. J., \&Houseworth, J. (2016). Is mathematical representation of problems an evidence-based strategy for students with mathematics difficulties? Exceptional Children, 1, 1-18. doi: 10.1177/0014402915625062

Landerl, K., Bevan, A., y Butterworth, B. (2004). Developmental dyscalculia and basic numerical capacities: A study of 8-9-year-old students. Cognition, 93(2), 99-125. doi: http://dx.doi.org/10.1016/j.cognition.2003.11.004

NTCM. National Council of Teachers of Mathematics (2006). Principles and standards for school mathematics. Reston, VA: Author.

Navarro-Cecilia, M. J., \& Navarro, J. I. (2015). Método de intervención matemática basado en la evidencia. Revista de Psicología y Educación, 10(2),113-126.

Passolunghi, M. C., Lanfranchi, S., Altoè, G. y Sollazzo, N. (2015). Early numerical abilities and cognitive skills in kindergarten children. Journal of Experimental Child Psychology, 135, 25-42. doi: http://dx.doi.org/10.1016/j.jecp.2015.02.001

Poff, B., McLaughlin, T. F., \& Derby, K. M. (2012). The effects of cover, copy, and compare with free time in math for elementary students with severe behavior disorders. Academic 
Research International, 2(2), 217.

Poncy, B. C., Skinner, C. H., y Jaspers, K. E. (2007). Evaluating and comparing interventions designed to enhance math fact accuracy and fluency: Cover, copy, and compare versus taped problems. Journal of Behavioral Education, 16, 27-37. doi: 10.1007/s10864-0069025-7

Ramirez, G., Gunderson, E. A., Levine, S. C., \&Beilock, S. L. (2013). Math anxiety, working memory, and math achievement in early elementary school. Journal of Cognition and Development, 14(2), 187-202. doi: http://dx.doi.org/10.1080/15248372.2012.664593

Rhymer, K., Skinner, C., Jackson, S., McNeill, S., Smith, T., \& Jackson, B. (2002). The 1minute explicit timing intervention: The influence of mathematics problem difficulty. Journal of Instructional Psychology, 29, 305-311.

Skinner, C. H., McLaughlin, T. F., \& Logan, P. (1997). Cover, copy, and compare: A selfmanaged academic intervention across skills, students, and settings. Journal of Behavioral Education, 7, 295-306.

Toll, S. W., Van der Ven, S. H., Kroesbergen, E. H., \& Van Luit, J. E. (2011). Execu-tive functions as predictors of math learning disabilities. Journal of Learning Disabilities, 44(6), 521-532.doi: 10.1177/0022219410387302

Van Luit, E. J, Van de Rijt, B., Araújo, A., Aguilar, M., Aragón, E., Ruiz, G., Navarro, J. I., Menacho, I., \& García-Sedeño, M. (2015). Test de evaluación de la competencia matemática temprana-informatizado (TEMT-i).Madrid: EOS. 